\title{
AEROSOL LAYERING CHARACTERIZATION NEAR THE GOBI DESERT BY A DOUBLE POLARIZATION LIDAR SYSTEM
}

\author{
Y. Zhao ${ }^{1}$, A. Boselli ${ }^{2}$, A.Sannino ${ }^{3}$, C. Song ${ }^{1,3}$, N. Spinelli ${ }^{3}$, X. Wang ${ }^{4, *}$ \\ ${ }^{1}$ BRIT, Beijing Research Institute for Telemetry, Beijing, P.R. China \\ ${ }^{2}$ CNISM and IMAA-CNR - C.da S. Loja 85050 Tito Scalo Potenza, Italy \\ ${ }^{3}$ CNISM and Dipartimento di Fisica - Università di Napoli "Federico II" - 80126 Napoli, Italy \\ ${ }^{4}$ CNISM and CNR-SPIN, - 80126 Napoli, Italy, *Email:wang@na.infn.it
}

\begin{abstract}
In order to carry out 4-D (space and time) analysis of the atmospheric aerosol distribution and to make a characterization of their properties and time evolution, a transportable multi-wavelength, Elastic/Raman scanning lidar system with angular scanning capability has been realized. The system uses a diode pumped Nd:YAG laser source, specifically designed for this device, and a receiving systems able to detect elastic signals at 355, 532 and $1064 \mathrm{~nm}$ and Raman signals at 386, 407 and $607 \mathrm{~nm}$. It also allows to perform aerosol depolarization measurements at both $355 \mathrm{~nm}$ and $532 \mathrm{~nm}$.
\end{abstract}

A first measurement campaign has been carried out in Dunhuang, North-West of China, in the region of the Gobi desert with the aims to study and characterize desert dust at source.

Optical properties of aerosol layers developing in the atmosphere have been analyzed and lidar data are discussed in terms of profiles of aerosol backscatter coefficient at $355 \mathrm{~nm}, 532 \mathrm{~nm}$, aerosol extinction coefficient at $355 \mathrm{~nm}$, aerosol depolarization ratio at $355 \mathrm{~nm}$ and $532 \mathrm{~nm}$ and water vapor mixing ratio. Depolarization ratio measured simultaneously at two wavelengths allowed also to study its dependence on the wavelength.

\section{INTRODUCTION}

Air pollution is a problem that affects for a long time the industrialized countries of Europe and more recently, with the increasing economical and industrial development, China's cities. Those last are characterized by a reduction of the visibility also in absence of special weather condition (e.g fog and rain) depending on high concentrations of particulate matter, often exceeding healthy limit values prescribed by the normative.

The pollutants resulting from human activities (related to vehicular traffic, domestic heating and industrial activity) and those related to natural sources (volcanic eruptions, forest fires, long range transport of mineral particles from desert regions) change the composition and physicalchemical characteristics of the atmosphere, thereby generating a clear reduction of visibility.

In large urban areas, atmospheric pollution is a serious problem recognized by the World Health Organization that highlighted the need to use resources and energy in the development of new methodologies for real time monitoring of atmospheric particles, in order to analyze its effect on air quality.

Advanced systems for environmental monitoring that use the lidar technology allow the real-time monitoring of suspended particles in the atmosphere and a characterization of their geometrical, optical and microphysical properties in space and time.

Lidar systems combining elastic and Raman observations are already successfully used in the aerosols tropospheric and stratospheric studies in the Asian region [1,2], especially during dust transport events, with the purpose to reveal the spatial and temporal distributions of dust and to study the ambient air quality.

In this paper, we describe the results of a measurement campaign performed in Dunhuang on 19-20 of August 2013 with the aim to study and characterize mineral aerosol directly at source. 
Measurements have been performed with a new lidar apparatus designed and implemented by CNISM (National Consortium of Italian Universities for the Physical Science of the Matter) and BRIT (Beijing Research Institute of Telemetry) in the frame of the AMPLE (Aerosol Multi-wavelength Polarization Lidar Experiment) project.

\section{METHODOLOGY}

The AMPLE lidar system has been designed to perform volume scanning of the atmosphere and to retrieve high quality $3 \mathrm{D}$ map of particulate optical properties and their time evolution.

The system uses a Nd:YAG diode-pumped laser source, specifically designed for this device, doubled and tripled in frequency and with a repetition rate of $1 \mathrm{KHz}$. The high repetition rate of the laser source can increase the detectable signal dynamic range allowing to monitor optically dense atmospheric layers (as cloud, and fog) and to make measurement with high speed scanning to follow rapidly variable phenomena.

The receiving system is able to detect both the elastic lidar returns at $355 \mathrm{~nm}$ and $532 \mathrm{~nm}$, the $\mathrm{N} 2$ Raman lidar echoes at $386 \mathrm{~nm}$ and $607 \mathrm{~nm}$ and the $\mathrm{H}_{2} \mathrm{O}$ Raman lidar echoes at $407 \mathrm{~nm}$. Moreover, polarization purity of laser line allows polarization measurements at both $355 \mathrm{~nm}$ and $532 \mathrm{~nm}$.

Each detected signal is acquired with a raw spatial resolution varying from $30 \mathrm{~cm}$ to $30 \mathrm{~m}$.

The lidar system has been calibrated through horizontal measurement method and iterative method for overlap function calibration. Multiwavelength channel calibration method has been implemented by using cirrus cloud, while an achromatic depolarizer has been used to calibrate the depolarization measurements [3].

Lidar profiles of aerosol backscattering coefficient at $355 \mathrm{~nm}$ and $532 \mathrm{~nm}$, aerosol extinction coefficient of $355 \mathrm{~nm}$, water vapor mixing ratio and aerosol depolarization ratio at $355 \mathrm{~nm}$ and $532 \mathrm{~nm}$ have been retrieved from lidar signals.

Their spatial and temporal distributions have been analyzed to study change in the aerosol optical properties measured in the observed layers. The Color Index (CI) expressed as:

$$
C I=\left(\log \left(\beta_{532} / \beta_{355}\right)\right) /(\log (355 / 532))
$$

has been evaluated in order to study the wavelength dependence of the backscatter coefficient.

Moreover, calibrated depolarization ratio simultaneously measured at two wavelengths allowed to study the aerosol depolarization dependence on the wavelength in addition to discriminate aerosol shape identifying the presence of non-spherical particles in the observed layers.

\section{RESULTS}

In Fig.1, the 24 hours continuous measurements performed in Dunhuang on 19-20 of August 2013 are reported in a color map of the Range Corrected Signal (RCS) that highlights several aerosol layer located below $4 \mathrm{~km}$ of altitude and a cirrus cloud in the range from 9 to $11 \mathrm{~km}$.

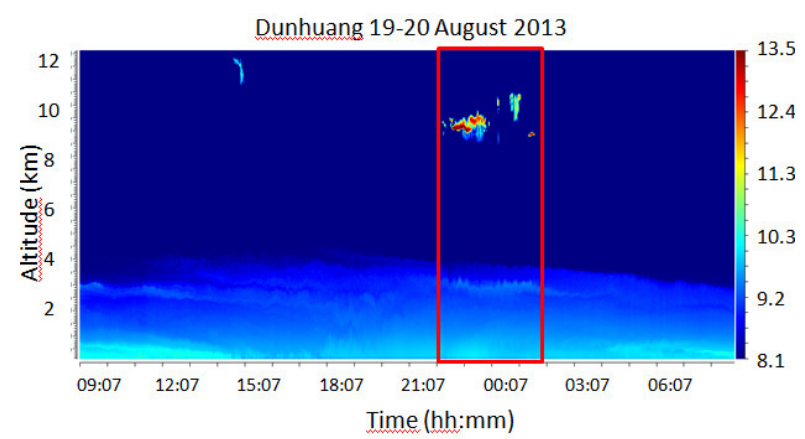

Fig:1 RCS color map of the 24 hours continuous measurements performed in Dunhuang on 19-20 of August 2013

A preliminary quantitative analysis of the data relative to the time interval between 22:00 UTC of 19 of August to 01:40 UTC of 20 (red line in the map) has been performed.

A very interesting feature develops after 22:00 UTC. It is spectacularly visible in the depolarization map of figure 2 .

It consists in an evident dynamical effects of rising of mineral particles from the soil giving rise to a stable layer at about $2 \mathrm{~km}$ of altitude. A layered structure of aerosol is also present up to about 5 kilometers of altitude. 


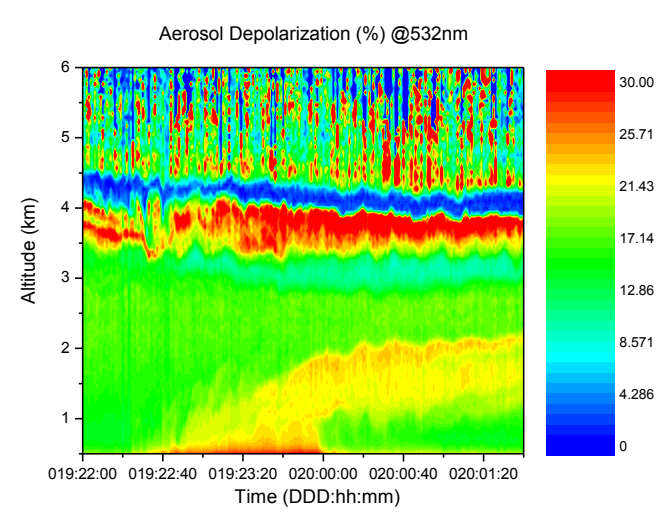

Fig:2 dynamical effects of rising of mineral particles from the soil giving rise to a stable layer at about $2 \mathrm{~km}$ of altitude

Data with a time resolution of 1 minute have been analyzed in term of aerosol backscattering coefficient $(\beta)$ and aerosol depolarization ratio $(\delta)$ at $355 \mathrm{~nm}$ and $532 \mathrm{~nm}$. The measured values of $\beta$ and $\delta$ resulted very close at the two wavelengths for cirrus, as expected from large particles, but they differs considerably for low altitude layers as a function of the different physical-chemical properties of particles in these layers.

This behavior is more clear in the scatter-plot of figure $3(a-b)$ showing the depolarization ratio at the two wavelengths. The values have been averaged in different atmospheric layers a) $0.5 \div 2$ $\mathrm{km}$, d) $8 \div 10.5 \mathrm{~km}$ relating to the observed aerosol signature in the lidar maps.

In figure $3 \mathrm{a}$ the values obtained in the first layer are shown. We observed a different dependence on the wavelength of the aerosol depolarization with time. In fact red point of the figure are related to the first 50 minutes of the measurement, before the observed rising of mineral particles. The different dimension of the particles could explain the observed different trend.

In the upper layer (figure $3 b$ ) where cirrus is present, data are clearly correlated and fitted by a line with slope 0.98 , indicating very close values between the two wavelength observed in the cloud.

Of course, a more detailed analysis of the optical and microphysical aerosol properties in these layers is required to better explain the observed results.

Data analysis of aerosol extinction and Lidar Ratio (LR) has been performed with a time resolution of $10 \mathrm{~min}$, in order to reduce statistical errors. Therefore, LR and Color Index (CI) have been analyzed in order to characterize aerosol and to define a particles classifications in each layer.
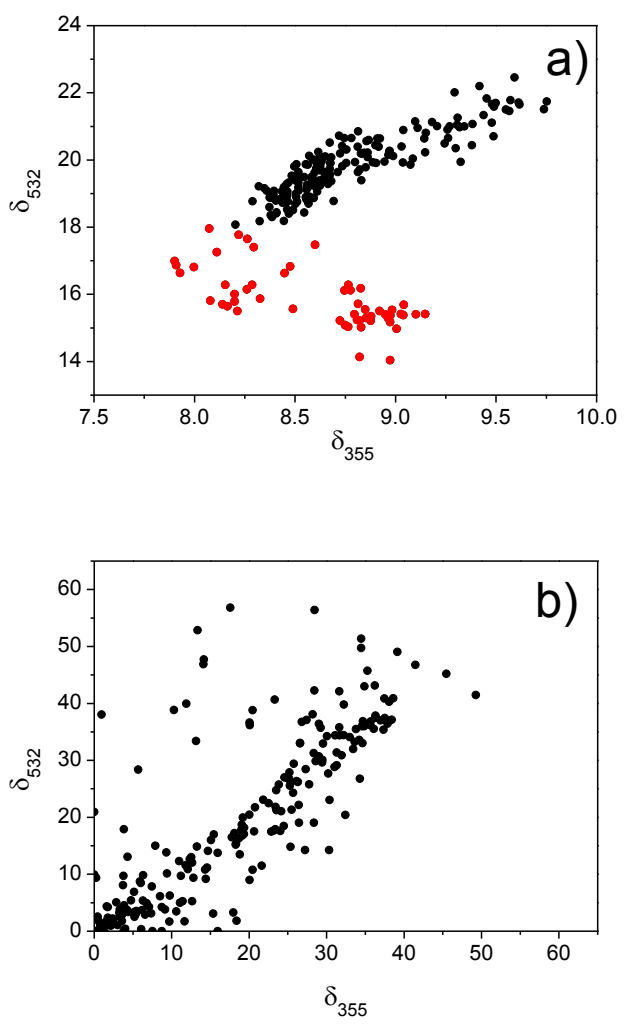

Fig:3 depolarization ratio at the two wavelengths in the atmospheric layers: a) $0.5-2 \mathrm{~km}$ and b) $8-10.5 \mathrm{~km}$

For cirrus we obtain values close to 0 for CI while a larger spread of LR values has been observed, indicating a change in the cloud properties with time.

Below $4 \mathrm{~km} \mathrm{LR}$ decreases with the altitude as the CI increases. A comparative analysis of the obtained values allowed identifying aerosol of different nature in each layer.

Water vapor mixing ratio showed the presence of a stable moist layer extending up to $4 \mathrm{~km}$, with a maximum at around $3 \mathrm{~km}$. Therefore, physical property of particles in this layer could be strongly influenced by this. 


\section{CONCLUSIONS}

An innovative lidar system has been developed and a first measurement campaign has been carried out in Dunhuang, North-West of China, in the region of the Gobi desert in order to study and characterize desert dust at source.

Elastic lidar signals have been analyzed in term of aerosol backscattering coefficient and aerosol depolarization ratio at $355 \mathrm{~nm}$ and $532 \mathrm{~nm}$. Moreover, from the Raman analysis the lidar ratio and the water vapour mixing ratio have been also determined. The results showed a layering with aerosol of different nature in each layer. Optical and microphysical properties of the aerosol have been studied.

\section{ACKNOWLEDGEMENT}

The financial support by the European Community through the ACTRIS Research Infrastructure Action under the 7th Framework Program, ACTRIS Grant Agreement n. 262254 is gratefully acknowledged.

\section{REFERENCES}

[1] J. Liu, Y. Zheng, Z. Li, C. Flynn, E.J. Welton, M. Cribb, Transport, vertical structure and radiative properties of dust events in southeast China determined from ground and space sensors, Atmospheric Environment 45 (2011) 6469-6480.

[2] Z. Chen, W. Liu, B. Heese, D. Althausen, H. Baars, T. Cheng, X. Shu, and T. Zhang, Aerosol optical properties observed by combined Ramanelastic backscatter lidar in winter 2009 in Pearl River Delta, south China, J. Geophys. Res. Atmos., (2013), 119, 2496-2510.

[3] X. Wang, A. Boselli, A. Sannino, C. Song, N. Spinelli, Y. Zhao, and C. Pan (2014) Calibration of Multi-wavelength Raman Polarization Lidar, EPJ Web of Conference, 89, 01002 (2015) . 